\title{
GEODETIC STEREO SAR WITH SMALL MULTI-DIRECTIONAL RADAR REFLECTORS
}

\author{
Martin Willberg' ${ }^{1}$, Christoph Gisinger ${ }^{1}$, Ulrich Balss², Thomas Fritz ${ }^{2}$, Michael Eineder ${ }^{2}$ \\ ${ }^{1}$ Chair of Astronomical and Physical Geodesy (IAPG), Technische Universität München (TUM), \\ D-80333 Munich, Germany \\ ${ }^{2}$ Remote Sensing Technology Institute (IMF), German Aerospace Center (DLR), \\ D-82230 Oberpfaffenhofen, Germany
}

\begin{abstract}
This paper evaluates the applicability and achievable SAR accuracy for octahedrons - a combination of eight corner reflectors with a common phase centre. In an experiment at the observatory in Wettzell from July to November 2015 these cost-efficient and mobile radar targets are measured with TerraSAR-X Staring Spotlight and High-Resolution Spotlight. Applying the geodetic stereo SAR concept, octahedrons are very robust for 3D-positioning through their backscattering in multiple directions. Using TBD size Octaeders, we achieve $3 \sigma$ standard deviations of about $3 \mathrm{~cm}$ for east, north and height components. For individual measurements in Staring Spotlight the standard deviation shows 1.4 $\mathrm{cm}$ in range and $3.2 \mathrm{~cm}$ in azimuth.
\end{abstract}

Index Terms - radar remote sensing, spaceborne radar, geodesy, synthetic aperture radar, stereo image processing

\section{INTRODUCTION}

During the last years several publications documented the progress in the accuracy of absolute range and azimuth measurements of highresolution SAR satellites [1, 2, 3, 4]. In the case of TerraSAR-X and TanDEM-X, the correction of atmospheric delay and geodynamic effects as well as external calibration allows an accuracy of $1.5 \mathrm{~cm}$ in range and $2.0 \mathrm{~cm}$ in azimuth [5]. Moreover, these observations enable absolute 3D positioning of similar accuracy [5]. These results have been demonstrated by measurements of corner reflectors (CR) with $1.5 \mathrm{~m}$ side length at the geodetic observatories Wettzell (Germany) and Metsähovi (Finland). These reflectors offer good Signal to Clutter Ratio (SCR) at resolution of $3 \mathrm{~m}$ in XBand, but they are large and expensive, and the stability against rain and wind makes them heavy and cumbersome for field usage.

In consequence, we have expanded our on-going research at Wettzell geodetic observatory. The aim is to test a different kind of reflector dedicated to positioning and exploiting the very high resolution of TerraSAR-X. The so called octahedrons are a combination of eight ordinary CRs arranged around a common phase centre, cf. Figure 1. Reduced to decimetre size, these octahedrons provide a significantly smaller Radar Cross Section (RCS) than classical CRs, but can offer several advantages by comparison to common CR.

Due to backscattering in multiple directions, the octahedrons can be measured from all available TerraSAR-X satellite passes which leads to a more robust 3D-positioning when using the stereo SAR concept [5]. Moreover, octahedrons are significantly less expensive and much lighter than classical CR and they are very mobile. The ones we used in our test have a dimension of $47 \mathrm{~cm}$, costs of about $40 €$ and weight less than a kilogram.

This paper discusses the usage of these octahedrons based on a dedicated experiment carried out at the geodetic observatory in Wettzell. In doing so the applicability of octahedrons, their pros and cons and the resulting accuracies for positions as well as measurements shall be tested.

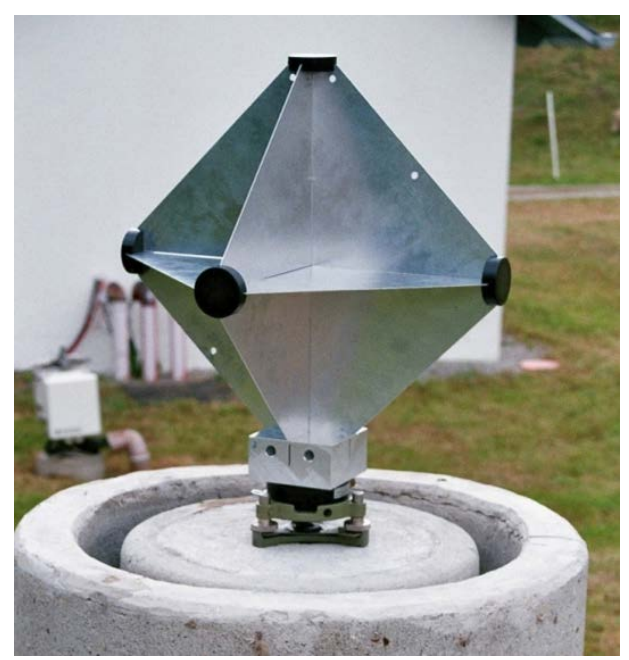

Figure 1: Octahedron reflector placed on a concrete pillar of the local geodetic survey network in Wettzell. 


\section{EXPERIMENTAL SETUP}

In summer 2015, four octahedrons (OT1-OT4) have been installed in Wettzell and measured with the TerraSAR-X satellite. From these four reflectors, two are mounted on geodetic measurement pillars (OT1, OT2, cf. Figure 1) whereas the octahedrons OT3 and OT4 are directly attached to ground. Their reference coordinates have been derived from on-site terrestrial survey within the local geodetic network, and can be considered to be better than $5 \mathrm{~mm}$.

All in all, 12 TerraSAR-X datatakes between July and November 2015 from different satellite orbits and modes are available (Table 1). Four datatakes have been captured in Staring Spotlight mode (ST) with the best possible resolution of $0.22 \mathrm{~m}$ in azimuth and $0.60 \mathrm{~m}$ in range. The remaining datatakes are High-Resolution Spotlight (HR) datatakes which provide less resolution in azimuth $(1.07 \mathrm{~m})$ [6]. Both ascending and descending acquisitions are involved.

Table 1: Overview on the available data acquisitions in Staring Spotlight (ST) and High-Resolution Spotlight (HS) mode.

\begin{tabular}{l|lll} 
Date & Mode & Geometry & $\begin{array}{l}\text { Incidence } \\
\text { angle }\end{array}$ \\
\hline 20150723 & ST & descending & $54^{\circ}$ \\
20150803 & ST & descending & $54^{\circ}$ \\
20150814 & ST & descending & $54^{\circ}$ \\
20150816 & ST & ascending & $34^{\circ}$ \\
\hline 20150825 & HS & descending & $54^{\circ}$ \\
20150905 & HS & descending & $54^{\circ}$ \\
20150916 & HS & descending & $54^{\circ}$ \\
20150927 & HS & descending & $54^{\circ}$ \\
20151008 & HS & descending & $54^{\circ}$ \\
20151019 & HS & descending & $54^{\circ}$ \\
20151030 & HS & descending & $54^{\circ}$ \\
20151104 & HS & descending & $45^{\circ}$
\end{tabular}

\section{STEREO SAR PROCESSING}

The data processing follows our concept named geodetic stereo SAR which yields global coordinates directly in the International Terrestrial Reference Frame (ITRF) 2008 and is described in full detail in [5]. Based on the TerraSAR-X L1B image products [7] the 2D-coordinates range and azimuth of the octahedrons are extracted from the radar images focused to zero-Doppler geometry by applying point target analysis (PTA). These raw measurements are corrected for atmospheric delay and geodynamic effects to enable accurate SAR positioning. Our correction scheme follows the conventions of the International Earth Rotation Service (IERS) [8] and can be found in [5]. Finally, the reflector coordinates are retrieved by solving the fully-linearized Range-Doppler equation system and using the corrected observations along with the TerraSAR-X Science orbit product. This geodetic least squares approach allows the combination of all observations in one single estimate and additional variance component estimation provides estimated standard deviations for range and azimuth in ST and HR. This is important, because the octahedrons show a higher noise in HR due to the smaller Signal to Clutter Ratio which results from the resolution.

These observation standard deviations, the standard deviation of the coordinate solution as well as the differences to reference coordinates provide the basis of our analysis. To simplify interpretation, the positioning results are transferred to a local NorthEast-Height (NEH) system.

For the available satellite passes of Wettzell given in Table 1, the three geometries combined in the joint-processing are visualized in Figure 2. Classical CR are limited to either ascending or descending satellite passes, while octahedrons remain visible in all available data acquisitions, cf. Figure 2. Thus octahedrons enable a better intersection geometry which directly affects the 3D position accuracy.

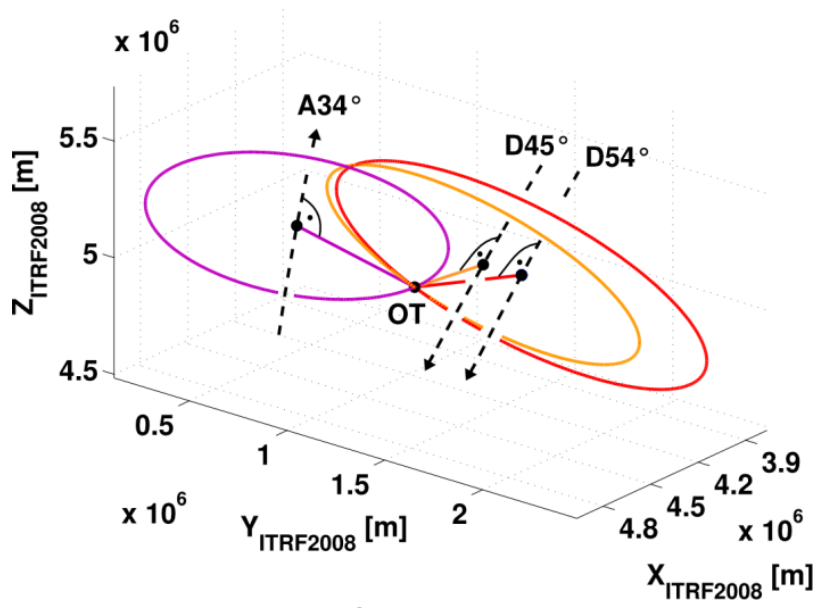

Figure 2: Visualization of available TerraSAR-X passes in Wettzell. Satellite zero-Doppler positions (black dots) and the measurements (range, azimuth) define oriented circles which intersect at the reflector position (OT).

\section{RESULTS}

The differences to the reference coordinates are visible in Table 2. They account for up to $\pm 1.7 \mathrm{~cm}$ in horizontal direction but have a constant offset in height of circa $5 \mathrm{~cm}$. The cause is not yet detected and could result from the satellite orbit, the 
Table 2: Octahedron positioning results in ITRF2008. $\Delta \mathrm{N}, \Delta \mathrm{E}$ and $\Delta \mathrm{H}$ are the differences to the reference coordinates, and $\sigma_{\mathrm{N}}, \sigma_{\mathrm{E}}$, $\sigma_{H}$ are the standard deviations $(3 \sigma)$ of the estimated positions. The values are given in north, east and height.

\begin{tabular}{l|ccc|ccc} 
& $\begin{array}{c}\Delta \mathrm{N} \\
{[\mathrm{cm}]}\end{array}$ & $\begin{array}{c}\Delta \mathrm{E} \\
{[\mathrm{cm}]}\end{array}$ & $\begin{array}{c}\Delta \mathrm{H} \\
{[\mathrm{cm}]}\end{array}$ & $\begin{array}{c}\sigma_{\mathrm{N}} \\
{[ \pm \mathrm{cm}]}\end{array}$ & $\begin{array}{c}\sigma_{\mathrm{O}} \\
{[ \pm \mathrm{cm}]}\end{array}$ & $\begin{array}{c}\sigma_{\mathrm{H}} \\
{[ \pm \mathrm{cm}]}\end{array}$ \\
\hline OT1 & -0.88 & 0.81 & 5.20 & 2.76 & 2.52 & 2.82 \\
OT2 & 0.41 & -1.72 & 5.56 & 3.06 & 2.49 & 2.76 \\
OT3 & 1.72 & -0.08 & 4.56 & 3.09 & 2.79 & 3.09 \\
OT4 & -0.19 & 0.24 & 4.39 & 2.85 & 2.25 & 2.67
\end{tabular}

scattering of the octahedron itself or the geometrical calibration. For further offset analysing first of all a new satellite orbit will be tested, because the revised version of the orbit affects the orbit calculation as well as the transformation of the satellite's mass centre to the phase centre of the antenna.

The estimated standard deviations $(3 \sigma)$ account for about $3 \mathrm{~cm}$ and represent the 95\% confidence interval of all measurements (Table 2). Through the acquisition geometry (cf. Figure 2) the confidence ellipsoid for all four octahedrons is almost similar to a sphere. For classical CR the estimated standard deviations are always dominated by an uncertainty in the coordinate orthogonal to range and azimuth [5].

The 2D standard deviations give the mean error of the solved positions to the measurements itself (cf. Table 3). The ST standard deviation for all octahedrons is about $1.3 \mathrm{~cm}$ and therefore $2-3$ times better than in HR. In azimuth one is able to accomplish about $3.2 \mathrm{~cm}$ in ST and $4.0 \mathrm{~cm}$ in HR mode. All in all the four octahedrons show similar behaviour, only OT4 differs with its surprisingly good range precision in High Resolution mode. For ST the achieved accuracy for these measurements is even similar to given SAR accuracy for corner reflectors of $1.5 \mathrm{~m}$ side length as used at the observatory in Wettzell.

Table 3: Results of the variance component estimation. Standard deviations of range $\sigma_{R}$ and azimuth $\sigma_{A}$ in Staring Spotlight (ST) and High-Resolution Spotlight (HR).

\begin{tabular}{l|ll|ll} 
CR & ST & & HR & \\
& $\sigma_{R}[ \pm \mathrm{cm}]$ & $\sigma_{\mathrm{A}}[ \pm \mathrm{cm}]$ & $\sigma_{\mathrm{R}}[ \pm \mathrm{cm}]$ & $\sigma_{\mathrm{A}}[ \pm \mathrm{cm}]$ \\
\hline OT1 & 1.32 & 2.97 & 2.91 & 3.79 \\
OT2 & 1.27 & 3.34 & 3.33 & 4.15 \\
OT3 & 1.44 & 3.53 & 3.44 & 4.10 \\
OT4 & 1.29 & 2.89 & 1.79 & 4.12
\end{tabular}

In comparison to results from classical CR [2, 4, 5] octahedrons contain a significant noise resulting from the background clutter. Based on the Radar Cross Section (RCS) of the octahedrons the SCR and the theoretical localization accuracy are calculated [9, 10]. Table 4 shows these accuracies when using TerraSAR-X and a background scattering of $-8 \mathrm{~dB}$. The better resolution in ST compared to HR leads to a higher SCR and a bigger weight in the estimation of ITRF2008 positions.

Table 4: Theoretical localization accuracy (noise) for octahedrons solely derived from the SCR. Values for TerraSAR-X with a background scatter of $-8 \mathrm{~dB}$.

\begin{tabular}{l|l|l} 
& $\begin{array}{l}\sigma_{\mathrm{R}, \text { OCTAHEDRoN }} \\
{[ \pm \mathrm{cm}]}\end{array}$ & $\begin{array}{l}\sigma_{\mathrm{A}, \text { OCtAHEDRON }} \\
{[ \pm \mathrm{cm}]}\end{array}$ \\
\hline HS & 3.03 & 5.40 \\
$\mathrm{ST}$ & 1.37 & 0.50
\end{tabular}

Even more detailed information is given in Figure 3 where the residuals of all measurements to the estimated values are shown. While the four ST acquisitions reveal a very high correlation among each other, the results differ for the HR datatakes. The high correlation in ST indicates that measurement errors in this mode do not primarily result from the reflectors. The higher noise for HR (cf. Table 3, 4) is clearly visible in Figure 3 for both range and azimuth.

When utilizing the four ST measurements only for $3 \mathrm{D}$ positioning, the $3 \sigma$ precisions amount already for about $4 \mathrm{~cm}$ in average. The adding of eight HR measurements has only a relatively small effect to the estimated precisions.

\section{CONCLUSIONS}

The presented results clearly show that SAR positioning with off sthe shelf TBD $\mathrm{cm}$ size octahedrons is possible in Staring Spotlight and High Resolution Spotlight with TerraSAR-X. Because of the small size, background clutter dominates the positioning error in HR which makes ST first choice for the high-precision measurements. To use octahedrons more effectively with HR the dimension should be increased.

For available measurements the 2D standard deviation for ST accounts for $1.3 \mathrm{~cm}$ in range and $3.2 \mathrm{~cm}$ in azimuth. The $3 \sigma$ standard deviations account for about $3 \mathrm{~cm}$ in north, east and height 

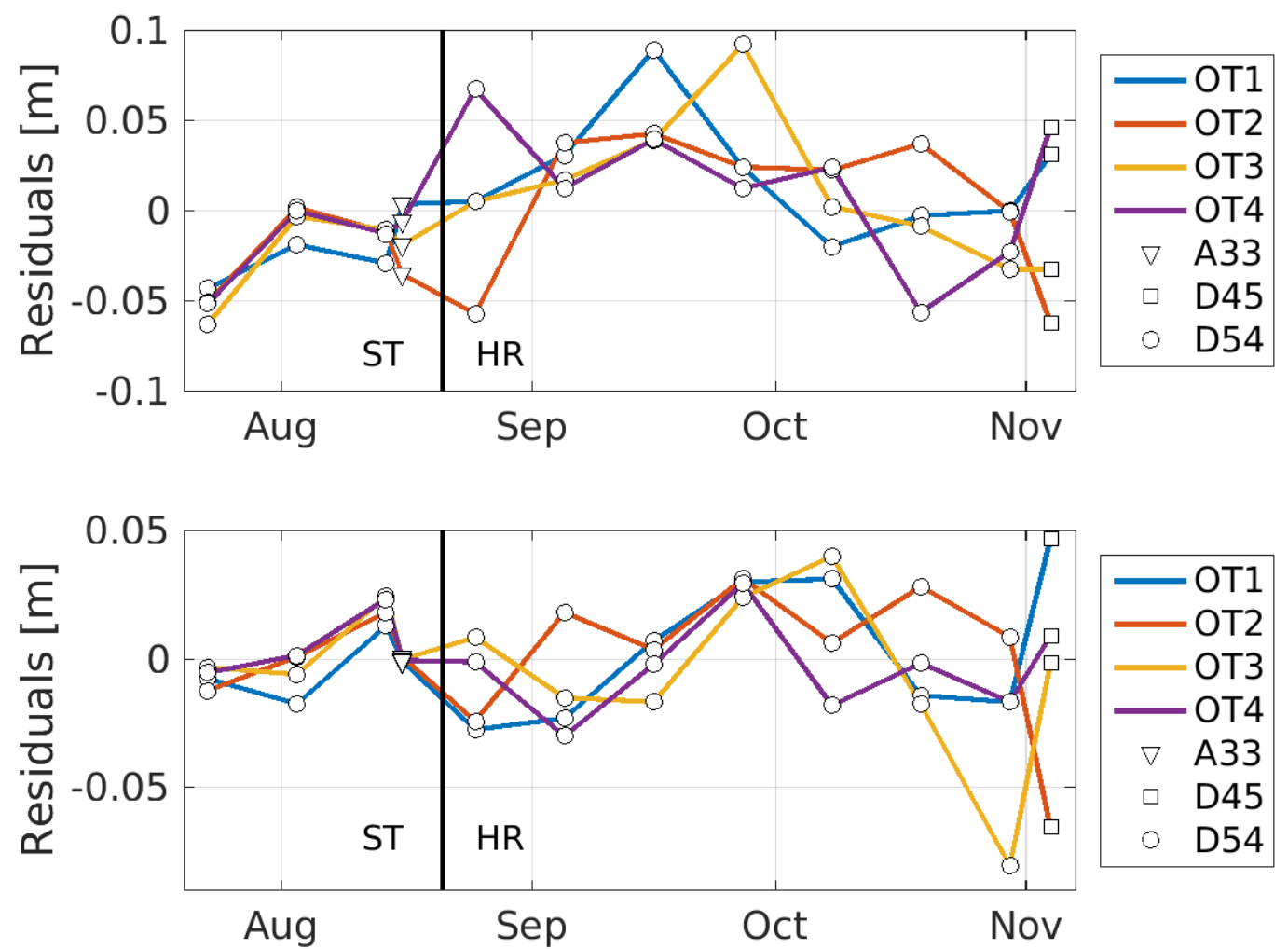

Figure 3: Observation residuals for the four octahedrons for azimuth (above) and range (below). The first four values are from the Staring Spotlight (ST) acquisitions, the following from High Resolution (HR). The different signs symbolize the associated geometry.

component, but the reason for height offset to reference coordinates is not yet detected.

Octahedrons show practical advantages due to small weight and size as well as robust 3D positioning. Compared to stereo imaging of CRs with $1.5 \mathrm{~m}$ side length, the double-sided measurement geometry in ST is even able to compensate the noise of octahedrons due to their smaller SCR. The high correlation in the residuals of range and azimuth for Staring Spotlight makes the data promising for differential evaluation.

\section{REFERENCES}

[1] A. Schubert, M. Jehle, D. Small, and E. Meier, "Mitigation of atmospheric perturbations and solid Earth movements in a TerraSAR-X time-series,” J. of Geodesy, vol. 86, pp. 257-270, 2011.

[2] M. Eineder, C. Minet, P. Steigenberger, X. Cong, and T. Fritz, "Imaging Geodesy - Toward Centimeter-Level Ranging Accuracy with TerraSAR-X," IEEE Trans. Geosc. Remote Sens., vol. 49, pp. 661-671, 2011.

[3] A. Schubert, D. Small, M. Jehle, E. Meier, "COSMOSkyMed, TerraSAR-X, and RADARSAT-2 Geolocation Accuracy after Compensation for Earth-System Effects,“ Proc. IGARSS 12, Munich, pp. 3301-3304, 2012

[4] U. Balss, C. Gisinger, X. Cong, R. Brcic, S. Hackel, and
M. Eineder, "Precise Measurements on the Absolute Localization Accuracy of TerraSAR-X on the Base of FarDistributed Test Sites," Proc. EUSAR $2014-10^{\text {th }}$ European Conference on Synthetic Aperture Radar, Berlin, Germany, 2014.

[5] C. Gisinger, U. Balss, R. Pail, X. Zhu, S. Montazeri, S. Gernhardt, and M. Eineder, "Precise Three-Dimensional Stereo Localization of Corner Reflectors and Persistent Scatterers With TerraSAR-X," IEEE Trans. Geosc. Remote Sens., vol. 53, no. 4, pp. 1782-1802, 2015.

[6] T. Fritz and M. Eineder, "TerraSAR-X ground segment basic product specification document,” TX-GS-DD-3302, v1.9, 2013. [Online]. Available: http://terrasar-x.dlr.de/

[7] T. Fritz et al., "TerraSAR-X ground segment Level 1b product format specification,” TX-GS-DD-3307, v1.3, 2007. [Online]. Available: http://terrasar-x.dlr.de/

[8] G. Petit and B. Luzum, Eds., IERS Conventions (2010), Verlag des Bundesamts für Kartographie und Geodäsie, 2010. [Online]. Available: http://tai.bipm.org/iers/conv 2010/conv2010.html.

[9] A. Freeman, "SAR Calibration: An Overview," IEEE Trans. Geosc. Remote Sens., vol. 30, no. 6, pp. 1107-1121, 1992

[10] P. Prats-Iraola, R. Schreiber, L. Marotti, S. Wollstadt and A. Reigber, „TOPS Interferometry With TerraSAR$\mathrm{X}$,“ IEEE Trans. Geosc. Remote Sens., vol. 50, no. 8, pp. 3179-3188, 2012 